\title{
GeoNotes: A Location-based Information System for Public Spaces 1
}

\author{
Per Persson, Fredrik Espinoza, Petra Fagerberg, Anna Sandin \& Rickard Cöster \\ http://geonotes.sics.se/
}

\subsection{Introduction}

The basic idea behind location-based information systems is to connect information pieces to positions in outdoor or indoor space. Through position technologies such as Global Positioning System (GPS), GSM positioning, Wireless LAN positioning or Bluetooth positioning, the system keeps track of where a terminal (and its user) is located in space. Via his terminal, the user is allowed to enter/upload information, to which the system automatically allocates a latitude-longitude coordinate. Later, the same user, or some other user, can access that information (again via their wirelessly connected terminals) when they enter the place. Although the digital information is stored on a remote server away from the actual location, the position technology and the mobile terminals give users the impression that information is actually "attached" to the place where the user is. In this way, location-based information systems create user experiences similar to those of post-its, graffiti and public signs and posters. In both cases, an information space is "superimposed" on indoor/outdoor space.

Quite a few systems have been working with this basic concept over the last five years within the fields of augmented reality, wearable and ubiquitous computing (Abowd, et al., 1997; Broadbent \& Marti, 1997; Burrell \& Gay, 2001; Cheverest et al., 2000; Caswell \& Debaty, 2000; Leonhardi et al., 1999; Marmasse \& Schmandt, 2000; Pascoe, 1997). Some have used goggles as access medium (Rekimoto \& Ayatsuka, 1998). Most, however, work with handheld devices that may not provide the same stunning and spectacular visual effects, but still preserve the basic functionality.

All the same, none of this research has directly addressed the social and navigational implications of location-based information systems. If we allow all users to author and access others' digital annotations without restriction, a rich information space will be created, but information overload will become a problem. In similar ways to the World Wide Web, we must design navigational tools to search, filter and sort information according to users' information needs. In contrast to the Web, however, location-based information will be superimposed on the real, physical world. Authoring and accessing information will not take place in offices and homes, but rather in public spaces, including streets, squares, pubs, public transportation, churches, cafés, galleries, malls and libraries. Potentially, the ways in which information is produced and consumed will be transformed by the spatial context.

\footnotetext{
1 Published in Höök, Kristina, Benyon, David \& Munro, Alan (eds.) (2002) Designing Information Spaces: The Social Navigation Approach, Springer, pp. 151-173.
} 
Equally true, peoples' understanding of (public) space will be affected by the overlaid information. We will describe a fully implemented system - GeoNotes - that takes a step in this direction.

First, we describe the functions of analog location-based information systems such as post-its, graffiti, signs and posters. Then we describe the interaction design of GeoNotes. ${ }^{2}$ Finally, we explain the technical implementation of the system.

\subsection{Analog location-based information systems in public spaces}

Location-based information systems are not a new phenomenon. Since the cavepaintings 30,000 years ago, humans have annotated space with imagery, figures and text, and human societies have developed technologies to support this, e.g., graffiti, posters, billboards, neon signs, and paper post-its. Although digital location-based information systems need not slavishly replicate the features of their analog counterparts, they may offer design inspirations.

On a physical level, analog annotation technologies adhere to surfaces in varying degrees. Glue, pins, fridge magnets, ink, and paint are perhaps the most common ways of accomplishing this, with different endurance performances. Some stay put for centuries (paint and carvings) while others wither and disappear quickly (messages on a sandy beach or ink in the rain). Some surfaces are somewhat resistant to annotation. Textile materials, for instance, are not well compliant with post-it notes. In order to impede annotations, some public space surfaces such as house façades, metro wagons and buses are even manufactured in anti-graffiti, easy-to-clean materials.

Analog annotation systems support different forms of media. Paper-based annotation systems, for instance, allows for text and still imagery. Neon signs and screen-based annotation systems support moving imagery in addition to that.

Sizing is pivotal since it determines the exactness of the placement of the annotation. A big poster, for instance, can be placed on a wall, but hardly on a mug or a piece of paper (cf. post-it notes). The smaller the annotation, the more exact its position in space.

On the other hand - and now we enter the communicatory dimensions of locationbased information systems - large-scale annotations have a perceptual salience that attracts more attention in public environments. Size at daytime and neon-lighting at night, augment the 'sensory radius' within which an annotation is perceptible to passersby. Small signs require careful attention to be detected. In some cases, a headline or an image captures our attention, but we have to approach the poster or sign in order to access its full content. In extreme cases, the overwhelming perceptual salience of a billboard or outdoor neon sign makes it difficult to steer clear of: it 'pushes' itself onto the passersby not unlike spam e-mails and directed marketing.

Since author and reader share the same spatial context, the message can refer to that context without loss of understanding, so called spatial deixis. For instance, attaching a post-it note to a piece of paper with the text "Peter, would you please make 10 copies of this?/Angie" is perfectly comprehensible since the position of the

\footnotetext{
${ }^{2}$ In Persson, et al. (2001) and Espinoza et al. (2001) we describe earlier versions of the system.
} 
annotation clarifies the reference of the word this. The more exact position of the annotation, the more authors can rely on deictic expressions. This, of course, also makes messages shorter and more economical.

It is also essential to point out that location-based annotations have many functions. Posters and signs may inform about events, activities, facts and authorities' decisions. But they may also persuade people to change ideologies or rethink their lives. Image- and text-based graffiti often makes political and philosophical statements. Commercial billboards and marketing encourage people to consume more or differently than they normally do. And classified advertisements connect buyers and sellers. Some annotations fulfill aesthetic functions, such as public art, paintings, and some graffiti. Finally, location-based annotations also operate as effective reminders to oneself and others. In many cases, it is more efficient to direct reminders to places than to points in time (via e.g., calendars and alarm clocks). A post-it note on the door, for instance, reminds me to bring my keys in the exact right moment (exiting the apartment before I slam the door locked).

In addition to the physical and communicatory dimensions, social aspects determine how people annotate space and how those annotations are produced. The assumed reader affects the contents of an annotation. Post-it messages between peers, family members and colleagues take place in a shared understanding of one another's preferences, sense of humor, personality, tasks and daily routines. By exploiting and alluding to this shared context, messages can be short and yet expressive. In public spaces, the potential reader of the message may not be entirely clear, which forces the author to deal with more general subjects. Still, many places are assumed to attract a certain clientele. In Stockholm, billboard producers can expect a Swedish audience; in a mall, one can expect shoppers; and on a university campus, students and teachers will be the most likely reader of your message. In addition, since many places physically restrict access to large groups of people, target audience can be clear (only family in my house, only students in the university library, only Nokia employees in Nokia facilities, only males in the gent's room). For sure, the placement of the annotation affects the subjects chosen and the opinions expressed.

To maximize visibility, annotations are often placed in passageways where it is known that many people (of the appropriate target audience) have to go through. Examples include entrances/exits of malls, stores and metro stations, as well as traffic intense gateways and portals into and out of cities.

If the author's understanding of the reader affects the annotation, the opposite is equally true. In particular, the degree of the authors' anonymity plays a role here, i.e. the extent to which readers can trace (or not trace) the unique identity of the author (Erickson \& Kellogg, 2000). If an author is reassured that her identity will be maintained secret, she will not be morally or legally accountable for opinions expressed or statements made. Such situations can be liberating for many annotators since one can express what one feels, bring taboos and political incorrectness to the surface, joke about forbidden phenomena and criticize authorities - all without social reprimands. On the other hand, yielding accountability may also encourage racist and sexist statements, invoking social stereotypes and leading the way for criminality and abuse of power. For what it is worth, anonymity tends to unleash the society's "unconscious". 
A softer form of anonymity involves anonymous signatures, in which the unique identity of the author is unknown but its repeated occurrence in several messages indicates the same source ("Mokaby was here").

With increasing risk of discovery - e.g., through witnesses and handwriting analysis - turf taggers, graffiti writers and anonymous poster publishers will presumably become less daring and subversive. In a fully non-anonymous situation, the author's identity is clearly recognized which makes him subject to potential public criticism, counterarguments or even legal charges. One may need to defend statements made and beliefs expressed.

Of course, making ones views, attitudes, personality and preferences known in a public setting is not something people generally avoid. On the contrary, it is striven for. Humans are social animals with deep going needs to show personal and social identity to others, and become recognized, accepted and respected for it. By articulating our attitudes to others, we "become" someone. By exhibiting what we think and are, we mark our group belonging in terms of ethnicity, gender, religion, class, social status, sports clubs, communities, interests groups, political parties, music taste, etc. (Willis, 1990). Political demonstrations, manifestations, performance, public posters, information flyers or 'legal' graffiti are ways in which people express their views not anonymously, but in a fully transparent and open way. In these cases, the author wants to be identified and recognized as something, in order to heighten the public's awareness concerning some issue. This relates not only to individuals but also to organizations, authorities, commercial players and interest groups. Public space is the most prominent arena to express what we are and stand for, not only through annotating space, but also through behavior, clothing, hairstyles, jewelry and other consumer goods. By indulging in such 'social exhibitionism' we hope not only to be recognized, but also that other people will give feedback in all sorts of ways, acknowledging our presence, identity and beliefs. Without some form of social feedback, opening up to the socio-public space will be a waste of energy. As we shall see shortly, allowing all users to express themselves in public space - as well as supporting feedback mechanisms on those expressions - have been leading design principles in the GeoNotes application.

If humans are social, this means not only that we express ourselves, but also that we are inquisitive about other people's lives, personalities, and background. Private and celebrity gossiping is one expression of this social curiosity. Sometimes this urge is channeled through voyeurism (e.g., illicitly glancing at people in a street café), sometimes not (e.g., approaching people in bars and starting to talk to them). We are curious of other people and public annotations provide a window into the lives and worldviews of others. A local bulletin board, for instance, may tell quite a lot about the neighborhood and its inhabitants. Much of the attraction and pleasures of public spaces stems from the reciprocal tension between social exhibitionism and curiosity: one can see and be seen at the same time (Goffman, 1963).

Finally, the growing commercialization of public space is a hotly debated political issue (Klein, 2000). There is concern that public space is taken over by commercial interests through messages and logos on billboards, buildings, streets, clothes, cars, and even in schools. Branding public space has become a multi-billion dollar industry. To become visible in public space requires a substantial amount of marketing resources. Why are some allowed to 'tag' the urban environment, but not 
others? Who and what forces are to decide about images, messages, logos and architecture in public space? What is the right balance between buyable and noncommercial public space? How can we provide space for alternative players and messages? Underlying this political discussion is the assumption that public annotations affect not only our preferences and beliefs, but also the ways in which we act and behave. Although GeoNotes is certainly not the single answer, it provides a technology that puts the question under new light. It may even offer a balanced compromise between commercial players and organizations like Reclaim-the-Streets. Let us see how.

\subsection{GeoNotes: Interaction Design}

\subsubsection{Mass-annotations vs. Professional Content providers}

Most digital annotation systems built so far, rely on content created by professional providers, such as art institutions, museums, tourist organizations, and business interests (Abowd, et al., 1997; Broadbent \& Marti, 1997; Cheverest et al., 2000; Caswell \& Debaty, 2000; Marmasse \& Schmandt, 2000). Relying on professional content providers, these systems run the risk of making the information formal, official and impersonal. Although some users are great fans of museums and art galleries, these domains are not part of the fabric of everyday life, and they do not involve friends, families, (potential) acquaintances or other interpersonal relationships. Moreover, professionally created information often tends to be 'serious' and 'utility oriented' in ways in which post-its, graffiti, tagging and posters are not. In those systems, the social, expressive, and subversive functions of analog annotation technologies - described above - tend to be overlooked.

Another problem with professional content is that information easily becomes static. With large information spaces, maintaining and updating information is expensive and time consuming when relying on a few content providers. Since many users (and many information seeking situations) insist on information freshness, such an approach will eventually become untenable.

An alternative to this is to let information space free, allowing it to grow, expand and develop with its users. Usage will change the organization of information space (Dieberger et al., 2000). Instead of making location-based information systems a vertical (one way) channel for information, by allowing and encouraging 'ordinary' users to provide, update, remove and comment information, the information space will become more of a horizontal communication medium between users. In this scenario, all users can leave highly expressive traces in the system (and in the geography) for others to see, which will create social awareness (Dourish \& Belotti, 1992). The information space overlaying physical space will become more social, reflecting the lives, concerns and social reality of the users in that space, rather than reflecting the views of some organization or authority (cf. Klein, 2000).

This idea fundamentally affected the design of GeoNotes. All users are allowed to author as much content as they want. All content is accessible to all other users. There 
is no mechanism by which the author can restrict reading access to oneself or others. It is fully transparent system. Thus, it differs from systems such as GPS enabled ComMotion system (Marmasse \& Schmandt, 2000) and the goggle system of Rekimoto \& Ayatsuka (1998). Although both of these systems allow and encourage users' annotations, these are mainly directed to oneself as reminders and documents. The social aspect is lacking.

This radical transparency is not only a way to create more social user experiences. It is also a highly politically design decision, allowing digital information space to channel voices of anyone in a fully democratic way. In contrast to urban space, which is commercially and legally regulated as to who is allowed to annotate, GeoNotes grants all users equal status. GeoNotes is thus the equivalent of legalizing graffiti, tags and posters, as well as forbidding anyone to make money on private property billboards. If you have access to a GeoNotes equipped mobile terminal, you have the same right to digitally 'tag' your neighborhood as any multi-billion dollar transnational company. In physical urban space, such anarchy would devastate the architectural space of the city, leading to decadence, vandalism, wreckage and violence. With GeoNotes the anarchy - which is digital - can co-exist with the regulated and ordered city space - which is physical. In their experience of urban space, citizens can choose the one or the other (or, preferably, both).

This anarchic approach to information space sets heavy design requirements on the GeoNotes system. As the responsibility to structure GeoNotes information space is transferred from the information providers/authors to the information readers, readers will have to be provided with powerful tools in order to find information that is meaningful, up-to-date and relevant to their situation. Before outlining our solutions to this (section 6.3.4), we need to describe how authors create content in GeoNotes.

\subsubsection{Authoring and Placing a GeoNote}

In order to promote (en masse) annotations, a basic user requirement is that authoring and placing should be quick and easy. A GeoNote should be neither difficult nor time-consuming to produce, and the interface has to allow such spur-ofthe-moment authoring in ergonomically uncomfortable and noisy mobile circumstances (cf. the Post-it note). At the same time, it should be filled with content that is meaningful and somewhat structured, in order to support readers' navigation of them. We settled for three fields (see Color Plate 7). 


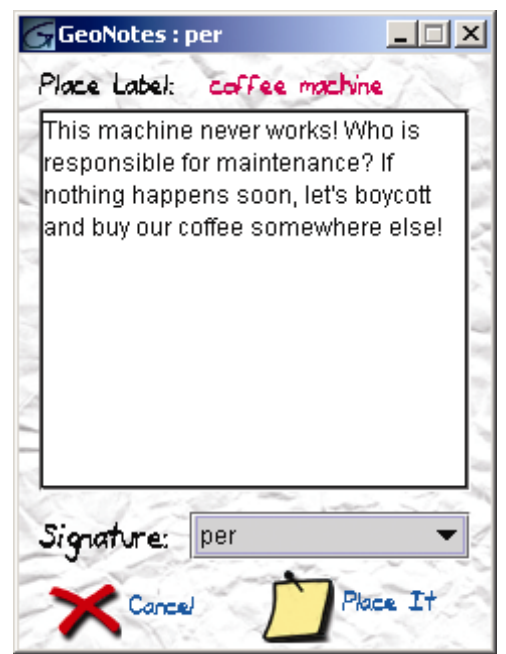

Color Plate 7. Authoring interface: Place label, message field, and the signature pop-up menu. User name is displayed at the top of the window.

The message field contains the content. Although sound files, images, and multimedia - in principle - could be part of the annotation, GeoNotes' current version supports text input only. GeoNotes makes use of the available text input mechanism of the terminal (keyboard on PCs, or pen-based input modes of PDAs).

In addition, the user has to choose a signature (below the message field). As mentioned, the anonymity/identity recognition of the author is an important regulator in public spaces. We wanted to support the same kind of processes in GeoNotes. On the one hand, users can choose to be anonymous to other people in the system. If the user, via the signature pop-up menu, chooses 'Anonymous' other users cannot trace him back to his GeoNote (see discussion below, section 6.3.4). On the other hand, users are allowed to create their own signatures in order to regulate their anonymity to the readers. In some cases the identity and contact information of the author is essential, e.g. in advertisements ('www.hem.passagen.se/anna', or 'Anna Sandin, telephone 070-633 15 07'). In others, e.g. digital graffiti, the identity of the creator needs to be recognized but not identified (e.g. 'SkateGirl'). These signatures, which can be easily created via the signature pop-up menu, are preserved in the system and available every time the user authors a new GeoNote.

The final action of the author is to allocate a specific position for the GeoNote. In the ultimate system, users would just hold their device in vicinity of a door, bookshelf, bench, tree or even a mug - and press 'place here'. This would be equally exact as attaching post-it notes to small-scale objects, and the users' understanding of here would be automatically understood by the system. For several reasons this is not likely to happen.

First, the accuracy of the author's positioning technology may be rather low. In the current version of GeoNotes, for instance, our W-LAN positioning gives quite rudimentary positioning data (even if refinement technologies such as base-station 
triangulation are employed) - see section 6.4. The radius of 'here' is rather large. Even if the author had a millimeter-precision positioning system, we have to expect other users to be less fortunate, depending on their terminals, type of wireless connection, software etc. (In fact, as a full fledged product, GeoNotes should allow the user to chose her positioning technology independent of the service.) In these cases, even if the author has accurately placed the GeoNote at, for instance, the handle on a door, a passing-by reader of that note may be equipped with a positioning technology with an accuracy as bad as 20 meters. Without some - by the reader understood - 'connection' between the author's intended place and the GeoNote, its message may become incomprehensible and meaningless (e.g., words referring to the spatial surrounding like this, here, and that).

For these reasons, a place label is required for each GeoNote. Place labels provide a semantic coupling between the message of the GeoNote and the place where the author intended it to be attached, more or less independent of the positioning technology. Even though authors and readers carry crude position precision, the place label describes - in natural language - where the GeoNote is thought to rest. Position technology acts as rangefinder; the place label as a focuser.

A place label system could be implemented in several ways. José \& Davies (1999) can be said to represent the formal approach in which the system creators systematize and organize space in addition to providing natural language labels (see also Burrell \& Gay, 2001; Caswell \& Debaty, 2000). By labeling rooms, corridors, elevators, buildings and specifying their spatial relations, the system administrators create a (formal) model of space. For instance, by defining inclusiveness, such a model can infer that 'Engineering building' is found within 'South Area', which in its turn is situated on 'University Campus'. Employing such tree-like, location-within-location model, the GeoNotes system would know the spatial surroundings of an annotation.

In the context of GeoNotes, there are two major problems with this approach. First, the effort and work required to categorize and maintain such a structure is enormous (imagine describing street corners, bus-stops, park benches, doors and desks on a global scale). Even more troublesome, however, is that place labels express the system administrators' view of space, not the users'. A description of a location in space can be quite subjective, depending on the users' situation, activity and even socio-cultural-personal background. Thus, to the greatest possible extent, users themselves should be able to provide the place labels.

In GeoNotes, place labels are provided by the author (before entering the authoring interface - see Color Plate 8). She can define a new place label in any way she likes in order to provide the maximum clarity in connecting the GeoNote to its intended position. We expect users to create all sorts of place labels, formal as well as personal (e.g., 'the ugly blue door'). 


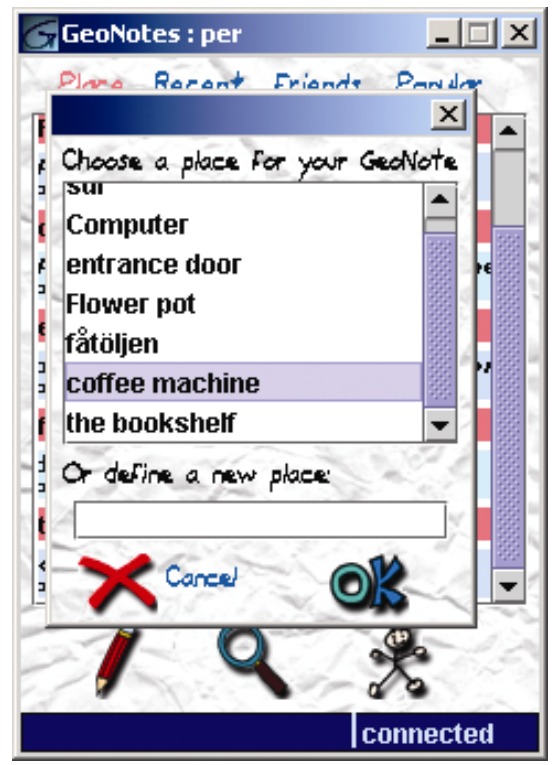

Color Plate 8. Choosing a place label.

In addition to creating new place labels, GeoNotes supports label sharing. Label sharing means that labels for a given place are stored in the system and may be reused by other authors of GeoNotes. When a user begins to author a GeoNote, the system collects all place labels that earlier authors have created (for that position) and displays them in a list (Color Plate 8). Although authors are free to create their own place label, they may save time if they can find an appropriate ready-made place label. Label sharing also allows users to get a sense of how other users appreciate and interpret a given place. How such traces of others influence authors' behavior and annotation activities is an interesting research problem that will have to be addressed in user studies.

Place label re-cycling also allows the system to sort out well-formulated and useful labels, from not-so useful or very personal labels. The list of available place labels will be sorted according to the number of times each label has been chosen (actually, only the top 10 labels will be displayed). This system encourages people to formulate useful place labels and creates a dynamic world of place labels that updates 'automatically' if the physical place changes.

This system also allows place labels to become more exactly positioned over time. By letting the user choose only among the 10 most used labels, poorly positioned labels will "disappear" or be recreated again by another user at a more exact location to gain new popularity. A well-positioned range of place labels will therefore grow dynamically. In addition, place label sharing could enhance the positioning precision of a user's device. If Mr. Good Position authors a GeoNote and creates a new place label, Mrs. Bad Position can later place her GeoNote on that place label. Since the position of that place label has a precision by far exceeding the capabilities of Mrs. 
Bad Position's device, she is able to 'take advantage' of Mr. Good Position's excellent position equipment.

Even more interestingly, the place label system is distinct from GeoNotes. Since place labels code real places that are not specific to the GeoNotes service, this means, in principle, that any location-based service can make use of them. The more usage the system is subjected to - through 'global' label sharing across services - the more likely it is that the place labels it contains will be relevant, accurate, and to the point for all services.

After these three steps, the author places the GeoNote (the 'Place-it' button in Color Plate 7). It should be noted that the system does not allow the author to restrict reading access to his GeoNote. As an analogy with public space posters, his annotation is fully visible to all passers-by.

Finally, it is worth pointing out that the author and the GeoNote must share position at the time of placing. Users can only place GeoNotes "here". Supporting authoring from remote sites was discarded for one major reason: we wanted this to be a location-based annotation system. A major purpose of GeoNotes is to investigate how location-based information differs from non-location-based information, e.g., the World Wide Web. Although a remotely authored GeoNote would have a position coordinate attached to it (chosen by the author via some map interface?), it would be difficult for the author to relate to the spatial context without being there. Instead, we wanted to investigate the consequences of a situation in which authors and readers of a message share the same space (but not time). ${ }^{3}$

\subsubsection{Social Feedback: Comments and Number of Readers}

GeoNotes provide a channel of expressing one's views, opinions, and concerns in public space. As mentioned above, however, it is equally central to get implicit or explicit feedback on the one's presence in public space: Was one's message received? How many received it? Was it recognized? Did it have the intended effects on the readers? Did people agree or disagree with the message?

If users of GeoNotes received answers to questions such as these, they would not only get a richer social experience from the system, we argued, but they would be encouraged to author many GeoNotes and fill the system with information. We provided a number of feedback mechanisms.

GeoNotes allows users to comment other people's GeoNotes. Color Plate 9 shows the reading interface of a GeoNote. Below the message and signature is indicated how many comments the note has and by clicking on the button the user is able to read those comments and to contribute with a new one. The comments interface operates in similar ways to normal discussion group windows, with the signature and a time stamp attached to each comment. As with any GeoNote, all comments are publicly accessible and commentators can choose signature and/or anonymity. The author can choose to receive notifications every time a comment is placed at one of her

\footnotetext{
${ }^{3}$ Instant messaging systems such as SMS and ICQ can thus be said to be the opposite of GeoNotes. In GeoNotes, authors and readers share space but not time, whereas SMS authors and readers share the same point in time (more or less), but not the same space.
} 
GeoNotes. The comment function allows dialogues between users similar to those of toilet graffiti discussions. In order to avoid removing other users' comments, GeoNotes authors are not allowed to remove their GeoNote if comments are attached to it.

Other feedback mechanisms are more implicit. For each GeoNote, the system allows the reader to give button-based feedback at the bottom of the view interface (Color Plate 9). 'Add to Favorites' means that the user 'picks it up', stores it locally on the client and has instant access to it even though the client is not connected to the GeoNotes server. It implies that the reader finds this information piece interesting. 'Blocking' a GeoNote means that the note will not again be visible for that reader. 'Blocking the author' means that all GeoNotes created by its author will become invisible to the reader in the future. These three feedback mechanisms are primarily tools for the reader to regulate information overload and filter out 'spam' GeoNotes as well as the senders of such spam. However, they are also used to promote social feedback to the author. GeoNotes allows users to access information about whether invited friends blocked their GeoNotes or added it to favorites (discussed in 6.3.4).

More implicit and anonymous feedback mechanisms are related to number of readers. Like any other user, authors can see how many readers any given GeoNote has had in the past (or per day).

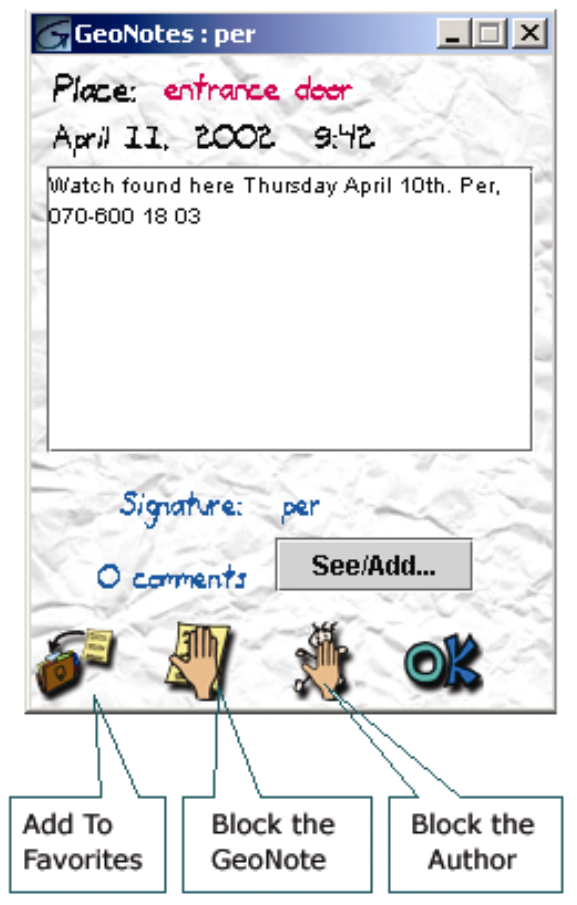

Color Plate 9. GeoNotes reading interface. This GeoNote has received no comments. 


\subsubsection{GeoNotes Access}

If we allow users to annotate digital space en masse and without restrictions, we may achieve a socially rich environment, but information space will become cluttered with unstructured information. How will users be able to find relevant and timely information in a system that has no central information designer? At a location with 5000 GeoNotes, how will the user be able to sort, filter and navigate this information space? If we allow GeoNotes to be 'pushed' to the mobile device when a user passes a place, how do we avoid irrelevant and disturbing notifications, and yet provide valuable and interesting GeoNotes? No location-based information system has addressed these navigational issues. For instance, the Graffiti system - based on Wireless LAN positioning with laptop computers as access terminals - enables users to annotate a university campus with virtual notes directed to friends and fellow students, but it provides little support for navigation (Burrell \& Gay, 2001).

GeoNotes supports both content-based and socially based access. In the contentbased approach, the user focuses on the information content; words in a document, images in a film, the meaning or the topic of a book. In socially based access, users search information based on the social context in which a given information piece is placed. Information seeking is then guided by questions such as:

- Who is the creator of this information?

- In what context was it created? Purpose? Genre? Organizational context? Cultural and historical context?

- Do I share taste, preferences, situation or worldview with the author?

- Is the author considered an authoritative, influential or otherwise powerful person?

- Has this information been recommended by someone whose expertise I trust?

- How many have read/seen this information and do I share taste, preferences, situation or worldview with these readers?

Such socially based information methods are much more common than we usually expect, not only in personal affairs but also in professional, commercial, cultural and academic life.

For content-based access, GeoNotes includes a search engine ("Searcher" in Color Plate ). The search engine supports full text search of all GeoNotes fields and provides instant index updating. In order to support socially based access, GeoNotes employs techniques of social navigation and collaborative filtering. Automatically capturing the behavior, actions and choices of unique users and then making this context information available to other users was an important requirement of the system. GeoNotes captures the following parameters:

- The identity of authors and commentators (unless their anonymous signature is used)

- The unique identity of readers

- The time stamp of reading, authoring and commenting 
- The unique identity of readers adding a GeoNote to favorites, blocking a GeoNote or blocking a sender in the reading interface (Color Plate 9) ${ }^{4}$

Although this information is available to the system, it is not, of course, immediately available to all users. As will be described in the following, GeoNotes balances carefully between promoting socially based access methods and social awareness on the one hand, and privacy and integrity on the other. Suffice to mention one general principle which governs some of the distribution of context information, namely that of friends. In general, the ways in which friends access films, music, documents, books, places, clubs, museums, events etc. are pivotal in our navigation in everyday life since we know their preferences, habits, and sometimes their intentions. Irrespective of whether a person shares the same taste as his friend or not, the ways in which the friend has authored and accessed information are crucial since he can relate his own preferences with those of his buddy. If I share the taste of my friend, I can imitate his access behavior. If I know that I do not share the taste of my friend, I know what information to stay away from. In both cases, the knowledge about the friends' preferences makes information about their access behavior valuable in the constant negotiation between me and information space.

In addition, since friends are trusted, users can be relatively sure that the context information made accessible to them will not be misused or taken advantage of. In similar ways to instant messaging systems such as ICQ, GeoNotes employs an invitation procedure in which each user accepts or declines requests from other users to become a friend. Only accepted users will have access to intimate information of my behavior in GeoNotes. The next section will describe exactly how this is solved in the system.

In addition to the parameter of content-based/socially based access, GeoNotes also supports a number of access modes. Since the situation and information needs of a mobile user will shift abruptly, it is important to provide 'pull', 'push' as well as mixed modes of access. In an active search, the user formulates his information need, enters a search string and retrieves matching GeoNotes. Here the user actively 'pulls' information. At the other extreme, 'push' systems 'throw' information at the user without any explicit request from the user's part. Notification-based systems such as telephones, messaging applications or reminder systems, force the user to interrupt his present activity and shift attention to the incoming message/information. If the information pushed is less relevant than the present activity, then disturbance is experienced. If the information pushed is experienced as more relevant than the present activity, the notification is experienced as efficient, making the user aware of something interesting that he otherwise would not have known. Such notifications may even create experiences of happy surprise.

In a mixed push/pull access mode, the user is paying attention to the search situation (he is not attending some other task), but he may not be actively searching. Since he may not be able to exactly formulate his information need, he explores the information space (Benyon \& Höök, 1997). In this more 'open' information search, the user is trying to come to grips with what he is looking for, and tends to be open for 'suggestions' from the environment. For instance, shopping for clothes initially

${ }^{4}$ For a technical description of how and where this information is stored, see section 6.4. 
tends to be a rather open question, but by browsing stores, shelves and prices, consumers' understanding of their needs becomes more focused.

GeoNotes, we reasoned, required navigation tools that would allow the user to shift easily between 'pull', 'push' and 'mixed' access mode. It is to the interfaces supporting these various access modes that we now turn.

\section{Mixed Access Mode: Main Window}

The primary purposes of GeoNotes main window are to provide the portal to reading, writing, searching and profile functionalities of the system (Color Plate 10). Equally important however, is to provide the user with an at-a-glance overview of GeoNotes in space so that he can take 'a closer look' if something appears interesting ('mixed' access mode). The main window lists all GeoNotes attached to the user's present position (except blocked GeoNotes - see above). The list of GeoNotes automatically updates as the user shifts location. The definition of 'location', and thus the number of GeoNotes displayed in the list, is dependent of the precision of the position technology carried by the user. In the current version of GeoNotes, which relies on Wireless LAN positioning, a location shift will occur when the user shifts base-station access.

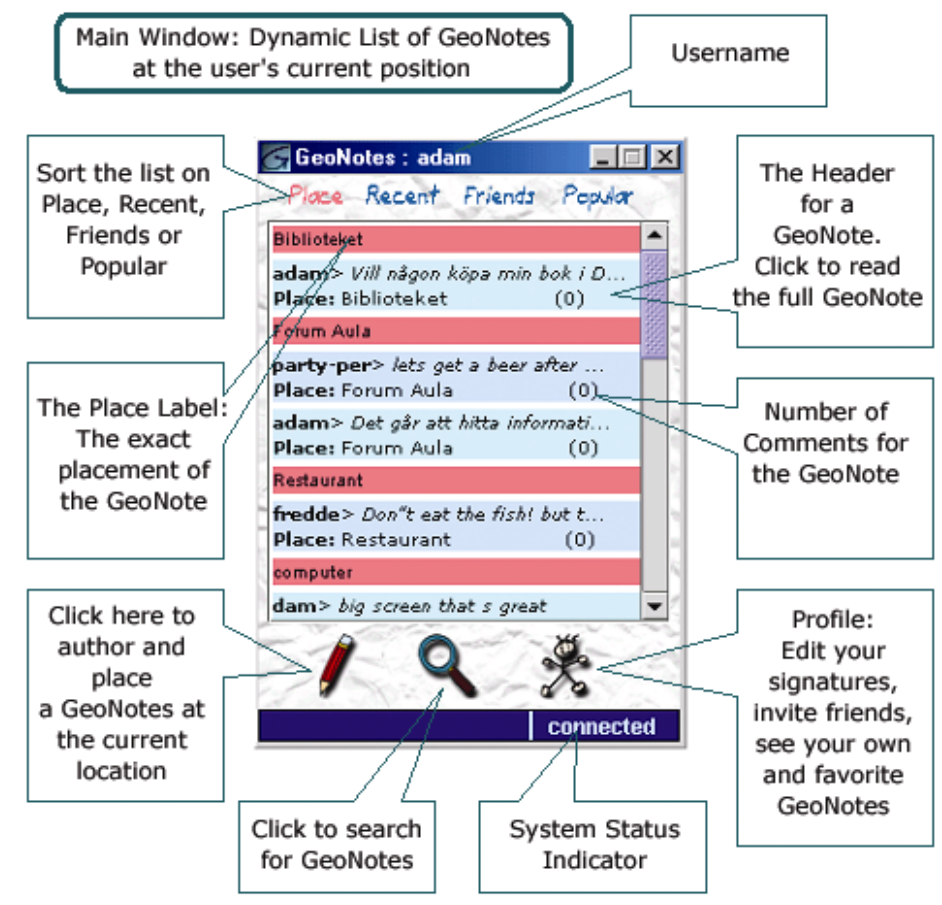

Color Plate 10. GeoNotes main window. 
Each GeoNote is listed as a header, containing the compressed version of its content (signature, the beginning of the message, place label and number of comments it has received). If something feels interesting, clicking on a header in the list brings the user to the view interface of the individual GeoNote (Color Plate 9).

Due to its expected length (we expect a great number of GeoNotes for each location), the list of GeoNotes needs sorting mechanisms in addition to mere scrolling. Sorting mechanisms, available at the top GeoNotes Main window, determine the order in which the GeoNotes are to be displayed in the list.

- Place (red tabs): Sorts all GeoNotes at current location according to place labels in alphabetical order. Good to use when entering a place and wanting to find GeoNotes on a particular object at the location.

- Recent (green tabs): Sorts the list according to when the GeoNotes were created. Recent GeoNotes appears first in the list.

- Friends (yellow tabs): Sorts the list with friends' GeoNotes first in the list. Each friend gets a tab. Then the users' own GeoNotes get listed, and then all others' GeoNotes.

- Popular (violet tabs): Sorts the list according to popularity. This is defined as total number of readings, divided by the number of days since the GeoNotes was authored. In order to prohibit authors to boost the popularity of their own GeoNotes, his own readings are excluded in this statistics.

\section{Pull access mode: the search window}

When users come to a location with hundreds or even thousands of GeoNotes, scrolling and browsing through the Main window list will not be a meaningful way to find things of interest. Even though sorting is employed, the number of GeoNotes may be overwhelming. Besides, users may be searching for kinds of GeoNotes not supported by the sorting buttons.

The search interface allows users to perform advanced searches by combining a text string ('Text Search') with one of several 'Search Options' (Color Plate 11).

- Signature: This search retrieves GeoNotes with a signature defined by the searcher.

- Friends: If people have agreed on becoming GeoNote friends with a user, that user can search for GeoNotes that one or several friends have written, read, added to favorites or commented.

- Popular: This search retrieves GeoNotes with high readings per day statistics.

- Comments: Retrieves GeoNotes with more than 5, 10 or 20 comments.

- Recommended: This search retrieves GeoNotes recommended by GeoNotes recommendation system. If we allow the system to keep track of how unique users behave, the system will be able to automatically cluster users with the same behavioral profile. If two users have read more or less the same GeoNotes, rejected the same GeoNotes and saved the same GeoNotes, we can expect them to share preferences even though 
they have never met. Within such a preference cluster of users, the system can recommend new GeoNotes on the basis of the actions of other users. The recommendations are thus based on automatic 'matching' of the usage history of users. This is the core feature of collaborative filtering techniques and recommendation systems (Konstan, et al., 1997; Svensson, et al., 2001). Since our system keeps track of usage data (reading) and provides channels for positive and negative feedback via 'save GeoNote', 'reject GeoNote' and 'reject author', it is well suited for collaborative filtering. Storage of user data and the clustering take place in the recommender module (see Color Plate 13).

- Date: Get GeoNotes with a certain age (Written Today, Written the last 7 days, Written the last 30 days).

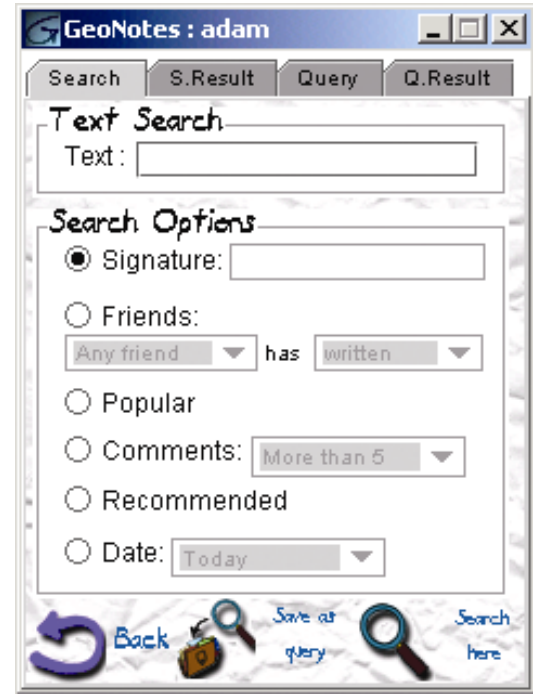

Color Plate 11. GeoNotes search interface.

The user is allowed to choose only one of these search options, but he may combine it with a text string. When the user has filled out the search fields, clicking the 'Search here' button retrieves the matching GeoNotes and displays them in a list (under the tab 'Search Results'). This list has the same structure as the list in the main window. In the future, we could also expect the results to be displayed on a finegrained map.

In the design of GeoNotes, we had several heated discussions of whether or not we should allow searches independent of the location of the user. Users could, for instance, generate global searches for GeoNotes or perhaps restrict searches to regions (via e.g., some map interface). In both cases, the user would be allowed to search for GeoNotes outside his perceptual space. For similar reasons as we discarded remote authoring of GeoNotes (see above 6.3.2), we finally settled for 'here-searches' only: 
We wanted to promote a spatially sensitive search paradigm, in which author and information seeker are positioned at the same place. Although GeoNotes are connected to a lat-long (latitude-longitude) coordinate, global searches tend to promote a system experience similar to that of the spatially contextless World Wide Web. In contrast, we wanted to profile GeoNotes as a location-based annotation system.

\section{Push access mode: Queries}

In a mobile setting, we cannot expect the user to stare at the GeoNotes main window as she moves through space. In contrast to a stationary user who pays attention to the computer screen all the time, the attention of a mobile user is typically occupied by other phenomena. The ergonomics of mobile usage makes it difficult to visually monitor a screen (walking in a busy urban environment, driving a car, paying a bus ticket). More importantly, however, the mobile user typically enters activities and situations in which there are so many more interesting things to do than monitoring GeoNotes information space: Sunday picnics with family, club-hopping with friends, skateboarding, shopping, observing street life, talking on the phone, admiring/studying urban and non-urban landscapes, tourism. In situations such as these, typically, the terminal will be placed in pocket or bag.

At the same time, many mobile users are still open for suggestions from the physical and social environment on how to enrich and improvise the present activity. GeoNotes' notification system allows the user to be notified of GeoNotes when he or she enters a new place. The notification consists of a sound signal and a pop-up window.

However, in order to avoid disturbance effects (discussed above), users must be able to create filters that only let through relevant GeoNotes. Since the situation and information need of the mobile user shifts abruptly, such a tool has to be easily configurable. At the same time, it has to be powerful enough to strike balance between filtering out too little and too much.

GeoNotes allows the user to create such filters through the search interface. Instead of 'searching here', however, the user is able to save his search as a 'query' (Color Plate 11). The user is asked to give his query a recognizable name, after which the query ends up in the user's query list (Color Plate 12). Queries have the same scope as searches, but constantly and continuously perform their search as users move through space. Whenever the user shifts location, the query scans the new location for appropriate GeoNotes and notifies the user if there is a match. All results are displayed in a list under the Query Result tab. In this way, queries operate like a mobile search engine, always 'accompanying' the user.

The user can add as many queries as she likes to her list. Via a checkbox she can deactivate any query (without deleting it) and quickly activate it again. She can also choose to deactivate the notification signal. With this box un-checked, results will be listed under Query results as normal, but no notification signal will be given. At the end of the day, the user may check if her movements through space "collected" any matched results, and she will be able to read those GeoNotes, even though she is offline and on another location. (Beside one's own GeoNotes and saved GeoNotes, this list is the only possibility to read GeoNotes remotely). 


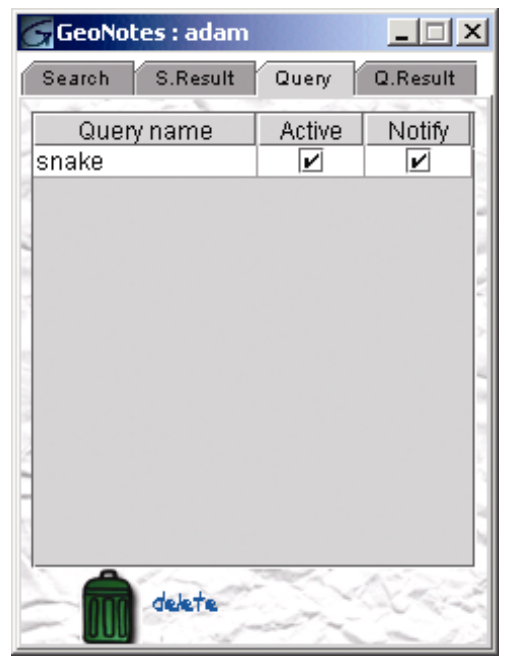

Color Plate 12. The list of Queries.

\subsection{Technical Implementation}

The GeoNotes implementation has partly been affected by the constraints of a forthcoming user study in the campus Wireless LAN network of Royal Institute of Technology $(\mathrm{KTH})$ in Kista. Since a meaningful study of GeoNotes requires a great number of users, utilizing the system over a longer period of time (at least a month), the KTH campus network provided a doable setup: at our disposal we will have 300 students equipped with Wireless LAN ready laptops. Although the area covered by the approximately 35 access points is limited, it is a neighborhood in which many students work, attend lectures, go for lunch, have coffee breaks, read, and study on a daily basis, often in accompany of their semi-mobile terminals. With little investment, this setup will, we hope, provide us with valuable, real-usage data of GeoNotes. We will encourage students to download the application and have the GeoNotes main window (Color Plate 10) on their desktop during their time in school. In fact, the interaction design described in the previous sections, was guided by a small-window approach, which would fit GeoNotes onto people's desktops beside their normal applications (like instant messaging software). This also enables us to run GeoNotes on Wireless LAN equipped PDAs, such as Compaq's iPAC.

Despite small area coverage and relatively crude positioning, Wireless LAN provides wireless access and position data in the same package. Measuring and cataloging the physical lat-long coordinates of the access points will provide each client with its position since the system keeps track of client-access point connections (via a configuring tool built by John Sevy at Drexel University). ${ }^{5}$ Technically, the latlongs of each access point are stored in a MySQL database (Color Plate 13).

\footnotetext{
${ }^{5} \mathrm{http}: / /$ gicl.mcs.drexel.edu/sevy/airport
} 


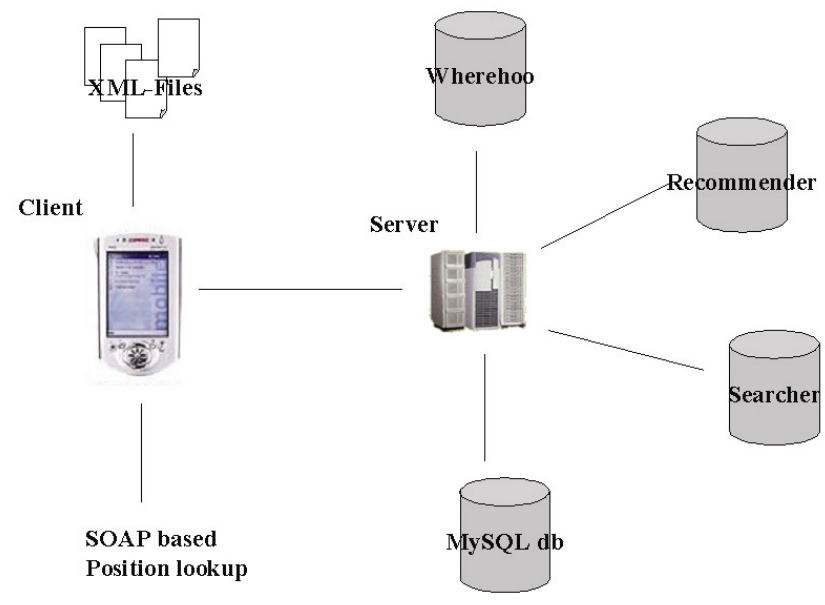

Color Plate 13. GeoNotes system architecture.

When the client connects to a new access point, the system detects its mac-address and gets its lat-long from the MySQL database. If this position data turns out to be too imprecise, we will have to experiment with triangulation or other refinement techniques (Castro, et al., 2001; Nord, et al., 2002).

Content and usage data for each GeoNote is stored in various databases. 'Searcher' stores message content, the comments, signature, and number of readings. It also keeps track of when a GeoNote was authored and the last time it was read. Searcher database hosts the content-based search methods described above.

'Recommender' stores users' actions in the system. The ways in which users save or block GeoNotes, block authors, read (others') GeoNotes or make comments, will affect the ways in which the Recommender generates user profiles. In principle, any action can be recorded. These profiles are used in two ways. On the one hand, they allow users to search for friends' actions in the search interface (Color Plate 11). On the other hand, recommender matches user profiles and generates clusters of users with the same preferences (also described above).

Searcher is a full text search engine that implements the Boolean and Vector Space models of information retrieval (Witten, Moffat, \& Bell, 1999). The text is indexed into an inverted file structure for fast query computation. Recommender is a general package for memory and model-based Collaborative Filtering. GeoNotes uses a variant of memory-based collaborative filtering described in (Breese, Heckerman \& Kadie, 1998), implemented using the nearest-neighbor search algorithm. Both Searcher and Recommender have been developed by Rickard Cöster. Since they are general engines, GeoNotes is one of several information domains to which they have been applied. Both packages are implemented in Java.

The client keeps track of the position and stores XML-files containing the user's profile information. This involves signatures, blocked GeoNotes, and GeoNotes friends. Since friends lists are mutual, there could be problem if one client suddenly 
was deleted or un-installed. For this reason, the server also keeps copies of users' friends-lists.

The server database stores information about passwords, friends and messages between users. Messaging could relate to inviting a user to become a friend, or accepting a request to enter the user's friends-list. If the user is not online, the server will store the message and forward it as soon as he comes online. The message function is also used for delivering messages to all users, e.g., invitation to upgrade to a new GeoNotes version. In addition to this, the server handles all requests from the client to the different databases. For instance, when a user enters a new location, the server retrieves the place labels and GeoNotes IDs for the present position from the MySQL database. With the ID in hand, the server then asks the Recommender and the Search databases for content and usage data.

As soon as GeoNotes users are equipped with more precise positioning technology, we will need a module that can scan an area for GeoNotes. Wherehoo, a location database developed at MIT Media Lab, provides precisely this functionality (Youll \& Krikorian, 2000). In Wherehoo, strings of text can be stored on lat-longs. It is possible to search over a range of lat-longs. In the future, GeoNotes IDs and place labels will preferably be stored in this database instead of the MySQL database.

To enable clients to access the GeoNotes server over the Internet and trough firewalls we implemented the communication between client and server using Simple Object Access Protocol (SOAP). This protocol carries XML encoded remote method invocations over HTTP. Another advantage of using SOAP is that we ensure that GeoNote clients can be implemented on any platform in any programming language. As long as the client sends the right SOAP messages to the server, the system will function correctly.

\subsection{Acknowledgments}

The authors would like to express their deep gratitude to Damien Bailly, Joanna Drapella, Tommy Gunnarsson, Tobias Larsson, Adam Lindström and Pawel Wiatr at Royal Institute of Technology for their work in the deployment of GeoNotes in the StockholmOpen.Net Wireless LAN. Most people at the HUMLE lab at SICS have provided fruitful and well-founded critique since GeoNotes' inception a couple of years ago. In particular, we would like to mention Elenor Cacciatore, Martin Svensson, Hanna Nyström, Markus Bylund and Kristina Höök. Jim Youll (MIT, Boston) has been generously helpful with our requests concerning Wherehoo. Kåre Synnes (CDT, Luleå) and Mikael Nehlsen (SICS, Kista) offered assistance in situations when it was most needed.

\section{References}

1. Abowd, G, Atkeson, C., Hong, J., Long, S., Kooper. R. \& Pinkerton, M. (1997) Cyberguide: A mobile context-aware tour guide, Wireless Networks, 3 (1997), 421-433. 
2. Benyon, D. \& Höök. K. (1997) Navigation in information spaces: supporting the individual, In INTERACT'97, Sydney: Chapman \& Hall.

3. Breese, Heckerman \& Kadie (1998) Empirical Analysis of Predictive Algorithms for Collaborative Filtering, Proceedings of the Fourteenth Conference on Uncertainty in Artificial Intelligence, Madison, WI.

4. Broadbent, J., and Marti, P. (1997) Location Aware Mobile Interactive Guides: usability issues, in Proceedings of the Fourth International Conference on Hypermedia and Interactivity in Museums (ICHIM97).

5. Burrell, J. \& Gay, G. (2001) Collectively Defining Context in a Mobile, Networked Computing Environment, in Extended Abstracts, CHI'01, ACM Press.

6. Castro, Chiu, Kremenek \& Munz (2001) A Probabilistic Room Location Service for Wireless Networked Environments, in Abowd, Brumitt \& Shafer (eds.) Ubicomp 2001: Ubiquitous Computing, International Conference Atlanta, Georgia, September 30 October 2, Berlin: Springer, p. 18-34.

7. Caswell, D. \& Debaty, P. (2000) Creating Web Representations for Places, Proceedings of HUC 2000, Bristol, England, pp. 114-26.

8. Cheverst, K., Davies, N., Mitchell, K., Friday, A. and Efstratiou, C. (2000) Developing a context-aware electronic tourist guide: some issues and experiences; Proceedings of the CHI'00, ACM Press, $17-24$.

9. Dieberger, A., Dourish, P., Höök, K., Resnick, P., and Wexelblat, A. (2000) Social Navigation: Techniques for building more usable systems, in Interactions, NovemberDecember issue, ACM, 2000.

10. Dourish, P. \& Bellotti, V. (1992) Awareness and coordination in shared workspaces. Proceedings of CSCW'92.

11. Erickson, T. \& Kellogg, W. (2000) Social translucence: an approach to designing systems that support social processes, ACM Transactions on Computer-Human Interaction, Vol. 7, Issue 1, 59-83.

12. Espinoza, F., Persson, P., Sandin, A., Nyström, H., Cacciatore. E. \& Bylund, M. (2001) GeoNotes: Social and Navigational Aspects of Location-Based Information Systems, in Abowd, Brumitt \& Shafer (eds.) Ubicomp 2001: Ubiquitous Computing, International Conference Atlanta, Georgia, September 30-October 2, Berlin: Springer, p. 2-17.

13. Goffman, E. (1963) Behavior in Public Places. Notes on the Social Organization of Gatherings, New York: The Free Press.

14. José, R., \& Davies, N., (1999) Scalable and Flexible Location-Based Services for Ubiquitous Information Access, In Handheld and Ubiquitous Computing, First International Symposium, Karlsruhe, Germany, September 1999.

15. Klein, N. (2000) No Logo: Taking Aim at the Brand Bullies, Picador.

16. Konstan, J.A., Miller, B.N., Maltz, D., Herlocker, J.L., Gordon, L.R., Riedl, J. (1997) GroupLens: Applying collaborative filtering to Usenet news, Communications of the ACM 40 (3), 77-87.

17. Leonhardi, A., Kubach, U., Rothermel, K., Fritz, A. (1999) Virtual Information Towers A Metaphor for Intuitive, Location-Aware Information Access in a Mobile Environment, Proceedings of the Third International Symposium on Wearable Computers (ISWC'99), San Fransisco, CA, USA, IEEE Press, 1999.

18. Marmasse, N \& Schmandt, C. (2000) Location-Aware Information Delivery with ComMotion, Proceedings of HUC 2000, Bristol, England, pp. 157-171.

19. Nord, Synnes \& Parnes (2002) An Architecture for Location Aware Applications, Proceedings of the Hawaii International Conference on System Sciences (HICSS-35), Big Island, Hawaii, USA.

20. Pascoe, J. (1997) The Stick-e Note Architecture: Extending the Interface Beyond the User, in Proceedings of IUI'97, 261-64. 
21. Persson, P., Espinoza, F. \& Elenor C. (2001), GeoNotes: Social Enhancement of Physical Space, Design-Expo at CHI'2001, Seattle, April, 2001.

22. Rekimoto, J., Ayatsuka, Y., (1998) Augment-able Reality: Situated Communication through Physical and Digital Spaces. In Proceedings of the $2^{\text {nd }}$ International Symposium on Wearable Computers, Oct 19-21, 1998, Pittsburgh, Pennsylvania, USA.

23. Svensson, M., Höök, K., Laaksolahti, J. \& Waern, A. (2001) Social Navigation of Food Recipes, Proceedings of CHI'01, ACM Press, 341-48.

24. Willis, P. (1990) Common Culture. Symbolic work at play in the everyday cultures of the young, Boulder: Westview Press.

25. Witten, I.H., Moffat, A., and Bell, T.C. (1999) Managing gigabytes: compressing and indexing documents and images ( $2^{\text {nd }}$ ed). Morgan Kaufmann, San Francisco, CA.

26. Youll, J. \& Krikorian, R. (2000) Wherehoo Server: An interactive location service for software agents and intelligent systems, Workshop on Infrastructure for Smart Devices How to Make Ubiquity an Actuality, The Second International Symposium on Handheld and Ubiquitous Computing, Bristol (UK), September 27, 2000. 